\title{
Inelastic Shape Changes of Silicon Particles and Stress Evolution at Binder/Particle Interface in a Composite Electrode during Lithiation/Delithiation Cycling
}

\author{
Hailong Wang ${ }^{1}$, Siva P V Nadimpalli ${ }^{2, *}$ and Vivek B Shenoy ${ }^{1, *}$ \\ ${ }^{1}$ Department of Materials Science and Engineering, University of Pennsylvania, 19104; \\ ${ }^{2}$ Department of Mechanical and Industrial Engineering, New Jersey Institute of Technology, \\ 07102; \\ ${ }^{*}$ Corresponding authors: \\ Dr. Vivek B Shenoy Email: vshenoy@seas.upenn.edu; \\ Dr. Siva P V Nadimpalli: Email: siva.p.nadimpalli@njit.edu
}

\begin{abstract}
Inelastic shape changes of Si particles and stress evolution at binder/particle interface was modeled using coupled diffusion-stress framework available in finite element software. A simple model that contains two spherical Si particles with and without the polymer binder film was used to represent the composite electrode. The particles were lithiated and delithiated at two different rates: onerepresenting a slow charging case which results in a uniform $\mathrm{Li}$ concentration throughout the Si particles and the other representing a fast charging condition which results in non-uniform lithium concentration within the spherical Si particles. The inelastic shape changes and associated contact forces predicted by the model are qualitatively consistent with experimental data. Further, the effect of binder mechanical properties and the binder fraction on the stress evolution in Si particles and at the binder/particle interface was calculated. The proposed model, although simple, can guide a battery design engineer to choose a proper binder, charge/discharge strategy, and binder fraction for a durable electrode design.

Keywords: plastic deformation of lithiated $\mathrm{Si}$, binder/particle interface stress, composite electrode model, Li-ion battery, stress-diffusion, finite element method
\end{abstract}




\section{Introduction}

Owing to its high lithiation capacity (3579 mAh/g), low discharge potential and low density, $\mathrm{Si}$ is considered to be one of the promising materials that could potentially replace graphite as negative electrode in secondary Li-ion batteries[1,2]. As a result Si-based composite electrodes have been the focus of recent studies[3-10].In general, the commercial lithium-ion battery electrodes are composites made with slurry consisting of electrochemically-active powder such as graphite or silicon, conductive carbon additives, and a polymer binder. The composite Si electrodes are typically made with a large fraction (typically 80-90\%) of micronsized crystalline Si particles held together by carboxymethyl cellulose (CMC) polymer binder[10]. The large volume expansion/contraction characteristics of $\mathrm{Si}$ during $\mathrm{Li}$ insertion/extraction reactions have been a major road block for the wide-spread use of Si-based electrodes. These volume changes not only lead to significant contact stresses between particles in composite electrodes but also induce stresses in the polymer binder. The stresses could cause fracture of active particles and delamination of binder from active material or current collector, both of which lead to isolation of active particles from current collector and hence loss of electrical network. This is a predominant capacity fading mechanism in practical electrodes[11,12]. Hence, understanding the mechanical behavior of composite electrode under electrochemical charge/discharge conditions is necessary to improve the cyclic capability of commercial composite electrodes.

Significant progress has been made recently towards understanding the basic mechanical behavior of lithiated Si. In situ measurements of stress evolution and fracture behavior in thinfilm Si electrodes during lithiation and delithiation was carried out by Guduru and coworkers[13,14] and Zhao et al.[15]. It was showed by Sethuraman et al.[13] that the lithiated Si electrode undergoes inelastic deformation at ca. $1.5 \mathrm{GPa}$ and this could lead to plastic dissipation and energy loss. Shenoy and Johari[16] showed by DFT calculations that the tensile modulus of lithiated Si decreases with Li concentration. Zhao et al.[17], from their computational study, argued that the inelastic deformation could help alleviate the fracture of electrode particles. It was observed that nanoscale $\mathrm{Si}$ particles can accommodate the large volume changes in $\mathrm{Si}$ without leading to fracture [18-20]. For example, Si nanoparticles with a diameter less than 150 nm did not undergo fracture during electro chemical cycling [21]. Gu et al.[22] reported the lithiation behavior of Si nanoparticles attached to and embedded in a carbon nanofiber and found 
that the aggregated Si nanoparticles show contact flattening upon initial lithiation. Similarly, Hertzberg et al.[23]reported the irreversible shape change of Si tube in nano-confined space during the first Li insertion/extraction cycle. Several groups [24-28] have proposed models to simulate the deformation behavior of Si during lithiation/delithiation cycles, but no attempt has been made to incorporate the large deformation plasticity of Si and binder mechanical behavior into a composite electrode model. The role of binder on the cyclic behavior of the electrodes was studied by several researchers [29]. It was observed that the Si-based composite electrodes made with conventional binder (polyvinylidene fluoride or PVDF) showed poor cyclic performance compared to CMC [30,31]. This is attributed to the mechanical integrity of the binder [29] and the interaction between polymer and active particles [31]. Though these studies enhanced our understanding of cycling performance of Si-based composite electrodes, very few studies have been conducted to measure the stress evolution in the composite electrode, e.g., [33] and none modeled the mechanical behavior of a composite electrode. Stress measurements on composite Si electrodes suggested that the stress response is cycling-history-dependent which could be due to irreversible shape changes in contacting Si particles [32]. The contact stresses generated in a composite Si electrode could exceed the elastic limit of both Si active material and polymer which leads to permanent deformation or shape changes of Si particles causing history dependent deformation.

The objective of this study is to propose a simple model to capture the mechanics of a typical composite battery electrode. As a first step towards this, lithiation/delithiation of two Si particles in contact were simulated with and without binder. Finite element calculations were performed by treatingSi particles as elastic-plastic material and polymer binder as a hyperelasticmaterial. Asystematic investigation is carried out(i) to understand the effect of charging/discharging rate on the deformation behavior (or inelastic shape changes) of aggregating Si particles, (ii)to understand how theinelastic shape changes of particles influence the stress fields and overall mechanical behavior of a composite electrode in the subsequent cycles, specificallythestress fields within Si particles and at the polymer binder/particle interface, (iii) to compare the model predictions with experimental observations, and (iv) to understand the effect of process parameters such as the content of polymer binder on the binder/active material interface stresses.. Though the model was simple, the predictions are qualitatively consistent with the experimental data available on the composite Si electrode coatings. 


\section{Methods}

Inelastic deformation and permanent shape changes in lithiated Si particles and evolution of interface stress between Si and polymer binder were simulated during lithiation/delithiation cycling using the finite-element package ABAQUS [33]. Two spherical Si particles with radius $R R=100 \mathrm{~nm}$ [22], (Fig. 1a-b), covered with a uniform layer of polymer binder, with thicknesst, are consideredas shown in Fig. 1c-d. For the sake of simplicity, the clearance between neighboring particles $d$ is assumed to be equal to the binder layer thickness $t$, with $t=0$ representing Si particles without binder (Fig. 1e). Axisymmetric finite element model of two Si particles with electrochemical and mechanical boundary conditions with and without binder is shown in Fig. 1d and 1e, respectively. Although the diffusion of Li in Si could be influenced by stresses in the particle [24,34,35], the coupling was not considered in this study as the shape changes of particles may be weakly dependent on this coupling. As a result,the diffusion of Li in Si was assumed to be driven by the gradient of concentration alone and was modeled with a constant Li diffusivity $D=10^{-16} \mathrm{~m}^{2} / \mathrm{s}[36,37]$. These particles are lithiated/delithiated by implementing a constant current and constant voltage (CC-CV) boundary condition. It should be noted that, in a composite electrode, prescribing a constant total current does not guarantee constant current (or flux) at each and every point on the surface of the individual particles. However, for simplicity we assumed a constant current (or flux) on the surface of the particles. Also, strictly speaking, a constant chemical potential resembles constant voltage; however, as the chemical potential in this study only depends on the concentration, prescribing a constant concentration of $\mathrm{Li}$ on the surface is equivalent to prescribing a constant voltage. Further, a crystalline Si particle when reacted with Li will undergo crystalline to amorphous phase change in the initial cycle[38,39]; it stays amorphous for the rest of the cycling history, if the potentials are maintained above $50 \mathrm{mV}$. Here, we did not model the crystalline to amorphous reactions and focused mainly on the lithiation of amorphous Si.

The lithiation-induced elastic-plastic deformation of Si particles was described by the elastic and perfectly plastic model [17]. The increment of the total strain is taken to be the sum of three contributions,

$d \varepsilon_{i j}=d \varepsilon_{i j}^{l}+d \varepsilon_{i j}^{e}+d \varepsilon_{i j}^{p}$ 
where $\varepsilon_{i j}^{l}$, $d \varepsilon_{i j}^{e}$, and $d \varepsilon_{i j}^{p}$ are the incremental strain components due to lithiation, elastic deformation, and plastic deformation, respectively. The volume ratio $(J)$ is proportional to the normalized Li concentration $(\bar{C}), J=1+\Omega \bar{C}$, where $\Omega=2.7$ fromthe $270 \%$ increase in the volume for the alloying reaction of $\mathrm{Li}$ with $\mathrm{Si}$ at full lithiation, i.e., $L i_{15} S i_{4}[1,10]$. The increment of the lithiation-induced strain can be expressed as a function of the normalized $\mathrm{Li}$ concentration $\bar{C}, \varepsilon_{i j}^{l}=\delta_{i j} \operatorname{Ln}[1+\Omega \bar{C}] / 3=\delta_{i j} \alpha \bar{C}$, where $\alpha=\operatorname{Ln}[1+\Omega \bar{C}] /(3 \bar{C})$ is the effective expansion coefficient.A normalized Li concentration was defined as $\bar{C}=C / C_{\max }$ where $\bar{C}=0$ for pure Si and $\bar{C}=1$ for fully lithiated Si (i.e., $\left.L i_{15} S i_{4}\right)$. $\delta_{i j}=1$ for $i=j$, and $\delta_{i j}=0$ for $i \neq j$. The increment of the elastic strain $d \varepsilon_{i j}^{e}$ obeys Hook's law $d \varepsilon_{i j}^{e}=\left[(1+v) d \sigma_{i j}-\right.$ $\left.v d \sigma_{k k} \delta_{i j}\right] / E$, whereYoung's modulus, $E$, and Poisson ratio, $v$,are Li concentration-dependent.A linear relation based on first-principles calculations by Shenoy et al. was used [16],

$E_{S}(\bar{C})=E_{0}+\bar{C}\left(E_{1}-E_{0}\right)$

$v_{s}(\bar{C})=v_{0}+\bar{C}\left(v_{1}-v_{0}\right)$

Where $E_{0}=150 \mathrm{GPa}$ and $v_{0}=0.21$ corresponding to pure Si (or $\bar{C}=0$ ) and $E_{1}=50 \mathrm{GPa}$ and $v_{1}=0.23$ corresponding to fully lithiated $\operatorname{Si}($ orfor $\bar{C}=1$ ). The incremental plastic strain $d \varepsilon_{i j}^{p}=\lambda S_{i j}^{p}$ was assumed to obey the classic J2-flow rule: the plastic yielding occurs when the von Mises equivalent stress, $\sigma_{e}=\sqrt{3 S_{i j} S_{i j} / 2}$ reaches the yield stress, $\sigma_{\mathrm{y}}$, of lithiated Si. The $S_{i j}=\sigma_{i j}-\sigma_{k k} \delta_{i j} / 3$ is the deviatoric stress. The scalar $\lambda$ can be determined at each increment by solving the boundary value problem. Recent studies indicate that amorphous electrodes such as amorphous Si or amorphous Ge can exhibit rate dependent behavior [40,41], which is not considered in the current work. The yield stress was also assumed to be a linear function of $\mathrm{Li}$ concentration, based on the plastic flow stress reported by Sethuraman et al.[13]

$\sigma_{y}(C)=\sigma_{y}^{0}+\bar{C}\left(\sigma_{y}^{1}-\sigma_{y}^{0}\right)$

Here, $\sigma_{y}^{0}=1.5 \mathrm{GPa}$ for $\bar{C}=0, \sigma_{\mathrm{y}}{ }^{0}=0.6 \mathrm{GPa}$ for $\bar{C}=1$.

The Li concentration and stress-strain fields were incrementally updated using an implicitly coupled temperature-displacement procedure in ABAQUS [33]. The lithiation-induced strain was simulated by a thermal strain approach by replacing temperature with the normalized concentration and the effective thermal expansion coefficient $\alpha$, which is a function of 
temperature (or concentration). Young's modulus, Poisson ratio $v$ and yield stress $\sigma_{\mathrm{y}}$ are defined as $\mathrm{Li}$ concentration (i.e., temperature)-dependent properties to be updated based on the current $\mathrm{Li}$ concentration (i.e., temperature). In a composite electrode, Li diffuses through the binder to reach the surface of Si particles, which is dependent on the binder type. In the current model, we assume that the binder has good ionic and electronic conductivities and prescribe flux directly on the particle surface. The polymer binder is electrochemically inactive; hence, a zero expansion coefficient was prescribed. The binder was assumed to be a Neo-Hookean hyperelastic material [42],

$W=C_{1}\left(I_{1}-3\right)+D_{1}(J-1)^{2}$

where $W$ is strain energy density function, $I_{1}$ is the first invariant of the unimodular component of the left Cauchy-Green deformation tensor, and $J$ is the Jacobian of the deformation gradient. $C_{1}$ and $D_{1}$ are material constants. For consistency with linear elasticity in the limit of small strains, it is necessary that $C_{1}=E_{b} / 4\left(1+v_{b}\right)$ and $D_{1}=E_{b} / 6\left(1-2 v_{b}\right)$, where $E_{b}$ and $v_{b}$ are Young's modulus and Poisson ratio of polymer binder, respectively, of the polymer binder. Young's modulus of dry PVDF binder and CMC binder is 0.65GPa [43] and 4GPa [31,44], respectively. We assume that Poisson ratio of dry CMC binder $v_{b}^{C M C}$ is sameas that ofdry PVDF binder $v_{b}^{P V D F}=0.32[45]$.

In section 3.1, lithiation/ delithiation process was simulated by imposing a constant current (CC) or constant flux of $\mathrm{Li}$ on the surface of the Si.The average concentration of lithiated state throughout the model is $0.5 C_{\max }$, where $C_{\max }$ is the theoretical capacity of $\mathrm{Si}$ at room temperature (i.e., $29.89 \times 10^{9}$ Coulomb $/ \mathrm{m}^{3}$ based on $3579 \mathrm{mAh} / \mathrm{g}$ ). The lithiatedand delithiated states were achieved for different dimensionless lithiation/delithiation rates $I R / D C_{\max }$, where $I$ is the current density for CC charge and discharge. To study the influence of lithiation/delithiation rate on the evolution of stress and particle shape, twodifferent rates were consider were considered: (i) $I R / D C_{\max }=10^{-3}\left(I=29.89 \times 10^{-3} \mathrm{~A} / \mathrm{m}^{2}\right)$ representing a slow charging case which results in a uniform Li concentration throughout the Si particles and (ii) $I R / D C_{\max }=10\left(I=298.9 \mathrm{~A} / \mathrm{m}^{2}\right)$ representing a fast charging condition which results in nonuniform lithium concentration.

In section 3.2, the experimental stress evolution reported on a composite Si electrode (average size $5 \mu \mathrm{m}$ )[32] is simulated with the CC boundary conditionsconsistent with the 
experiments, i.e., charging rate of $C / 50$ and the discharging rate of $C / 100$ [32].The dimensionless lithiation and delithiation rates corresponding to these are $I R / D C_{\max }=$ 0.116 and 0.058 , respectively. Lithiation process was simulated by imposing a constant fluxof Lion the surface of the Si particle with charging rate of $C / 50$ for 3 hours; In delithiation process, a constant delithiation flux with discharging rate of $C / 100$ was imposed on the Si surface for 6 hours. A volume ratio of binder to spherical Si particleof 0.07 is prescribed in the model (i.e., Fig.1),i.e., $\frac{\phi_{b}}{\phi_{S i}}=\left(\left(1+\frac{t}{R}\right)^{3}-1\right)=0.070$, matching with the experimental values[32]. In addition to comparing the simulation data with experimental data, the influence of binder content on the contact stress and binder/particle interface stress is investigated by varying the volume ratio of binder to Si $\left(0.023<\phi_{b} / \phi_{S i}<0.115\right)$. Finally, two different binders(CMC and PVDF) were considered to understand the effect of binder modulus on the binder/particle interface stresses during electrochemical lithiation/delithiatoin process.

\section{Results and Discussion}

\subsection{Influence of Charging Rate on the Evolution of Stress Field and Particle Shape}

Fig. 2 shows the evolution of von Mises stress contours, contact forces, and aspect ratio of contacting $\mathrm{Si}$ particles under slow and fast lithiation/delithiation rates.The contact force $\left(\mathrm{N} / 4 \mathrm{R}^{2}\right)$ is defined as the sum of the reaction forces, $\mathrm{N}$, due to the mechanical constrains normalized by cross-section area, $4 R^{2}$. Note from Fig. 2a that when the lithiation is carried out at slow rates (i.e., $I R / D C_{\max }=10^{-3}$ ), particles expand uniformly without being subjected to any stresses; however, when the particles come in contact with their neighbors, significant amount of stresses will be generated at the point of contact (red contour),which can exceed the elastic limit at very low lithium concentrations during the very first lithiation process(indicated as L1-0.01in Fig. 2a, where L1 represents the first lithiation cycle and 0.01 represents the Li concentration $\bar{C}$ ). In contrast to Fig. 2a, when particles are lithiated rapidly, i.e., fast charging with $I R / D C_{\max }=$ 10 , stresses (beyond yield point) will be induced due to both non-uniform distribution of lithium concentration and particle-particle contact force as shown in Fig. 2b. Hence, energy dissipation due to the contact induced plastic deformation of particles could contribute to the energy losses that occur in the battery during first charge/discharge process. 
As the particles push against each other causing stresses beyond yield, they undergo permanent shape change which can be observed from the particles at the end of delithiation (shown as D1-0.01 in Fig. 2a, where D1 represents the first delithiation cycle). Figs. 2c and 2d show the evolution of particleshape and the contact force between them as a function of average lithium concentration during slow (solid curve)and fast (dashed curve) lithiation/delithiation processes. When the two particles are in contact with each other at the beginning of the lithiation, the free radiusof particle $R_{f}$ (where no particle contact occurs) and radius at contact point $R_{C}$ (defined in Fig. 2a and 2b) are equal to $R$ (initial particle radius). However, as the lithiation progresses, $R_{f}$ expands freely but $R_{c}$ remains constant due to constraint from the contact force, which causes the permanent shape change of Si particle from a sphere to a barrel type shape (as in Fig.2a and 2b). Note from Fig. 2c that, under slow charging conditions, if the particles are lithiated to same state of charge in subsequent cycles, most of the plastic deformation and the irreversible shape changes will take place in the first cycle. This is evident from the fact that the contact force (the blue solid curve in Fig. 2d) evolved nonlinearly in the first cycle but almost nonexistent for majority of the subsequent cycling except at the end of lithiation in each cycle when the particles come in contact. Hence, the permanent shape changes of particles influence the stress evolution in the subsequent cycles. The evolution of $R_{f}$ and $R_{c}$ (the red dashed curves in Fig. 2c) in fast charging case is slightly different from that of slow charging case due to the additional inhomogeneous lithiation induced plastic deformation. Also, during the fast charging process the contact force, Fig. 2d, does not increase monotonically with lithiation; it increases with lithium concentration initially, reaches a peak at $\bar{C}_{a v g} 0.45$, and decreases with further lithiaiton; the peak contact force is lower than that of slow charging case. This non-monotonic evolution and lower magnitude of peak contact force can be attributed to the fact that the particle surface softens relatively quickly in fast charging case due to increased lithium concentration at the surface causing lower contact force. Another key point to note is that the peak contact forcein fast charging case is highest during the initial lithiation/delithiation cycle, decreases during subsequent cycles, and completely vanishes after 10 lithiation/delithiation cycles (only 3 cycles are shown in Fig. 2d). This observation has some practical implications, i.e., if one manages to charge/discharge the electrodes to the same level everytime, the contact induced mechanical stresses will decrease within first few cycles and minimiz mechanical damage to some extent. 
The evolution of contact force and corresponding electrode stresses for the intermediate charge/discharge rates will be in between the two extreme cases discussed above.

\subsection{Influence of Elastic Properties and Content of Binder on the Contact Stress and Binder/Particle Interface Stresses}

Fig. 3a shows the stress response of a spin-cast composite Si electrode during first four lithiation/delithiation cycles from experiments conducted by Sethuraman et al.[32]. The electrode was lithiated to same state of charge in every cycle for the first four cycles.As explained earlier, the model did not simulate crystalline to amorphous phase changes of particles, hence the predictions are comparable to experimental data from second cycle onwards. In the absence of any other mechanical damage to the electrode microstructure, which was true in this experiment, the contact force evolution due to particle-particle contact predicted by the proposed model in Fig. 2 qualitatively agrees withthe evolution of compressive stress in Fig. 3a. The evolution of tensile stress in discharging cycles isprimarily attributed to the stresses due to surrounding binder which was not modeled in Fig.2. To mimic the experiments more closely, a thin layer of polymer binder (with CMC properties) is modeled between Si particles (see Fig.1d). Figs. 3b and 3c show the predicted evolution of contact stress and binder thickness between $\mathrm{Si}$, consistent with experiments. Note that the evolution of peak contact force for Si/CMC electrode in Fig. 3b is similar to that observed in Fig. $2 d$ and it isconsistent with thetrends observed in experimental data (Fig. 3a)[32], though there is some discrepancy in the magnitude of stress values. The discrepancy is expected as themeasured stress shown in Fig. 3a are not the stresses between two individual Si particles, but they are thickness averaged stresses. Further, the model assumes the silicon particles to be well defined spheres and did not represent the constraints on the particles exactly as in experiments.It is interesting to note that, in spite of its simple nature, the peak contact stress trend predicted by the model agreed very well with the experimental observations.

Note from Fig.3c that the contact forces arising due to expansion of Si particles can lead to significant amount of compression(as high as 86\%) in the binder layer which could cause binder damage during the first lithiation process. The permanent shape change of lithiated Si particles as seen in Fig. 2 will occur even in the presence of binder layer between particles (Fig.

3b-c), and this shape change willpotentially prevent binder damage in the subsequent cycles by lithiating the electrode to same state of charge as in Fig. 2d where the contact stress will either 
remain or reduce. Hence, by lithiating the electrodes to a constant state of charge (or lithiation level) could be a good strategy to minimize the stresses in the binder and the associated mechanical damage. It can also be noted that a stiffer binder, i.e., CMC, which is relatively stiffer compared to PVDF, will induce larger contact stresses and hence will influence the mechanical damage process significantly.

The normal/shear stress components at the interface were obtained by extracting the stress components (in global coordinate system)at the interface from the deformed configuration and transforming theminto the local coordinate system aligned with normal/tangent vectorsat the interface. This was done for varying time and position;Fig. 4 shows that the maximum normal stress and shear stress at the particle/binder interface, which are potential driving forces for debonding process, could reach to critical levels during the first lithiation/ delithiation process. The inset showsthe normal and shear stress distribution at the CMC binder/particle interfaceat the maximum and minimum lithiation level.Note that the maximum values of interface normal and shear stresses evolve with cycling as a result of shape changes in the particles. For example, peak tensile normal stress increases slightly and peak shear stress decreases with cycling, because of evolving contact forces observed in Fig. 3. Also, the binder properties have significant effect on the interface stresses. Note, however, that the effect is not so strong during the compression process as the peak compressive normal stress is almost same for both binders; however the peak tensile stress is significantly different. As a result, stiff binder requires strong bonds between binder and particles to prevent interface damage as the binder with a higher tensile modulus leads to higher stress for the same level of lithiation in the Si particles.

One of the important process parameters in battery manufacturing is the binder content. It is necessary to optimize the fraction of binder that improves durability but does not increase the dead weight to battery. In this context, it is important to understand the effect of binder content on the interface stresses which govern the mechanical failure of the electrode. Figure 5shows the effect of binder content on the evolutions of maximum normal and shear stresses at the binder/particle interface for CMC binder/Si particle. It can be noted that the peak normal stress at the interface, which is the primary component that could cause debonding, decreased with binder content and peak tensile normal stress almost becomes zero for binder/particle volume ratios beyond $V_{b} / V_{S i}=0.115$. A similar trend is observed for the interface shear stresses as well. Hence, capacity fading due to binder mechanical degradation can be reduced by increasing the 
binder content, but it decreases the overall capacity of the battery due to extra inactive binder. The results presented in this work are in agreement with the observations of the experiments (32, 33), and the proposed model, although simple, can guide a battery design engineer to choose a proper binder, charge/discharge strategy, and binder fraction for a durable electrode.

\section{Conclusion}

Axisymmetric finite element model of two spherical Si particles with and without polymer binder is considered as a model to represent composite electrode. Inelastic deformation and permanent shape changes in lithiated Si particles and evolution of interface stress between Si and polymer binder were simulated during lithiation/delithiation cycling using coupled stressdiffusion formulation available in ABAQUS finite element software.The lithiated and delithiated states were achieved for two different dimensionless lithiation/delithiation rates: (i) $I R / D C_{\max }=$ $10^{-3}$ representing a slow charging case which results in a uniform Li concentration throughout the Si particles and (ii) $I R / D C_{\max }=10^{3}$ representing a fast charging condition which results in nonuniform lithium concentration.

When particles were lithiated at very slow rates to allow for uniform concentration of lithium, the stresses in particles were primarily induced due to contact; however, when particles were lithiated rapidly, plastic deformation occurred due to both non-uniform distribution of lithium concentration and particle-particle contact force. Hence, energy dissipation due to the contact induced plastic deformation of particles could contribute to the energy losses that occur in the battery during first charge/discharge process. It was observed that the plastic deformation and the irreversible shape changes can be limited to initial lithiation/delithiation cycle if the particles are lithiated to similar state of charge in the subsequent cycles. The peak contact forcein fast charging case is highest during the initial lithiation/delithiation cycle and decreases during subsequent cycles consistent with the experimental observations on a composite Si electrode by Sethuraman et al. [32]. Hence, the permanent shape changes of particles influence the stress evolution in the subsequent cycles.

It was observed that the maximum normal stress and shear stress at the particle/binder interface, which are potential driving forces for debonding process, could reach to critical levels during the first lithiation/ delithiation process. As expected the particle/binder interface stresses depend on the properties of the binder, a stiffer binder leads to larger interface stresses. As a 
result, stiff binder requires strong bonds between binder and particles to prevent interface. An increase in the binder content reduces the stresses in the binder and at the interface. Hence, capacity fading due to binder mechanical degradation can be reduced by increasing the binder content, but it decreases the overall capacity of the battery due to extra inactive binder. The results presented in this work are in agreement with the observations of the experiments (33). The proposed model, although simple, can guide a battery design engineer to choose a proper binder, charge/discharge strategy, and binder fraction for a durable electrode.

\section{Acknowledgement.}

The authors gratefully acknowledge support of this work by the National Science Foundation (DMS-0914648), and the Department of Energy EPSCoR Implementation Grant (DESC0007074). SPVN would like to acknowledge the support of New Jersey Institute of Technology through the faculty startup grant.

\section{References}

1. Obrovac, M. N. \& Christensen, L. 2004 Structural Changes in Silicon Anodes during Lithium Insertion/Extraction. Electrochem. Solid-State Lett.7, A93-A96.

(doi:10.1149/1.1652421)

2. Kasavajjula, U., Wang, C. \& Appleby, A. J. 2007 Nano- and bulk-silicon-based insertion anodes for lithium-ion secondary cells. J. Power Sources. 163, 1003-1039.

(doi:10.1016/j.jpowsour.2006.09.084)

3. Mazouzi, D., Lestriez, B., Roué, L. \& Guyomard, D. 2009 Silicon Composite Electrode with High Capacity and Long Cycle Life. Electrochem. Solid-State Lett.12, A215-A218. (doi:10.1149/1.3212894)

4. Key, B., Bhattacharyya, R., Morcrette, M., Seznéc, V., Tarascon, J. M. \& Grey, C. P. 2009 Real-time NMR investigations of structural changes in silicon electrodes for lithiumion batteries. J. Am. Chem. Soc.131, 9239-9249. (doi:10.1021/ja8086278)

5. Liu, W.-R., Yang, M.-H., Wu, H.-C., Chiao, S. M. \& Wu, N.-L. 2005 Enhanced Cycle Life of Si Anode for Li-Ion Batteries by Using Modified Elastomeric Binder. Electrochem. Solid-State Lett.8, A100-A103. (doi:10.1149/1.1847685)

6. Buqa, H., Holzapfel, M., Krumeich, F., Veit, C. \& Novák, P. 2006 Study of styrene butadiene rubber and sodium methyl cellulose as binder for negative electrodes in lithiumion batteries. J. Power Sources161, 617-622. (doi:10.1016/j.jpowsour.2006.03.073) 
7. Beattie, S. D., Larcher, D., Morcrette, M., Simon, B. \& Tarascon, J.-M. 2008 Si Electrodes for Li-Ion Batteries-A New Way to Look at an Old Problem. J. Electrochem. Soc.155, A158-A163. (doi:10.1149/1.2817828)

8. Li, J., Lewis, R. B. \& Dahn, J. R. 2007 Sodium Carboxymethyl Cellulose: A Potential Binder for Si Negative Electrodes for Li-Ion Batteries. Electrochem. Solid-State Lett.10, A17-A20. (doi:10.1149/1.2398725)

9. $\quad$ Chen, L., Xie, X., Xie, J., Wang, K. \& Yang, J. 2006 Binder effect on cycling performance of silicon/carbon composite anodes for lithium ion batteries. J. Appl. Electrochem.36, 1099-1104. (doi:10.1007/s10800-006-9191-2)

10. Obrovac, M. N. \& Krause, L. J. 2007 Reversible Cycling of Crystalline Silicon Powder. J. Electrochem. Soc.154, A103-A108. (doi:10.1149/1.2402112)

11. Liu, G., Zheng, H., Simens, A. S., Minor, A. M., Song, X. \& Battaglia, V. S. 2007 Optimization of Acetylene Black Conductive Additive and PVDF Composition for HighPower Rechargeable Lithium-Ion Cells. J. Electrochem. Soc.154, A1129-A1134. (doi:10.1149/1.2792293)

12. Lee, J. H., Paik, U., Hackley, V. A. \& Choi, Y. M. 2006 Effect of poly(acrylic acid) on adhesion strength and electrochemical performance of natural graphite negative electrode for lithium-ion batteries. J. Power Sources161, 612-616.

(doi:10.1016/j.jpowsour.2006.03.087)

13. Sethuraman, V. A., Chon, M. J., Shimshak, M., Srinivasan, V. \& Guduru, P. R. 2010 In situ measurements of stress evolution in silicon thin films during electrochemical lithiation and delithiation. J. Power Sources195, 5062-5066.

(doi:10.1016/j.jpowsour.2010.02.013)

14. Nadimpalli, S. P. V., Sethuraman, V. A., Bucci, G., Srinivasan, V., Bower, A. F. \& Guduru, P. R. 2013 On Plastic Deformation and Fracture in Si Films during Electrochemical Lithiation/Delithiation Cycling. J. Electrochem. Soc.160, A1885-A1893. (doi:10.1149/2.098310jes)

15. Zhao, K., Tritsaris, G. A., Pharr, M., Wang, W. L., Okeke, O., Suo, Z., Vlassak, J. J. \& Kaxiras, E. 2012 Reactive flow in silicon electrodes assisted by the insertion of lithium. Nano Lett.12, 4397-4403. (doi:10.1021/nl302261w)

16. Shenoy, V. B., Johari, P. \& Qi, Y. 2010 Elastic softening of amorphous and crystalline LiSi Phases with increasing Li concentration: A first-principles study. J. Power Sources195, 6825-6830. (doi:10.1016/j.jpowsour.2010.04.044)

17. Zhao, K., Pharr, M., Vlassak, J. J. \& Suoa, Z. 2011 Inelastic hosts as electrodes for highcapacity lithium-ion batteries. J. Appl. Phys.109, 016110. (doi:10.1063/1.3525990) 
18. Chan, C. K., Peng, H., Liu, G., McIlwrath, K., Zhang, X. F., Huggins, R. A. \& Cui, Y. 2008 High-performance lithium battery anodes using silicon nanowires. Nat.

Nanotechnol.3, 31-35. (doi:10.1038/nnano.2007.411)

19. Takamura, T., Ohara, S., Uehara, M., Suzuki, J. \& Sekine, K. 2004 A vacuum deposited Si film having a Li extraction capacity over $2000 \mathrm{mAh} / \mathrm{g}$ with a long cycle life. J. Power Sources129, 96-100. (doi:10.1016/j.jpowsour.2003.11.014)

20. Kim, H., Han, B., Choo, J. \& Cho, J. 2008 Three-dimensional porous silicon particles for use in high-performance lithium secondary batteries. Angew. Chemie - Int. Ed.47, 1015110154. (doi:10.1002/anie.200804355)

21. Liu, X. H., Zhong, L., Huang, S., Mao, S. X., Zhu, T. \& Huang, J. Y. 2012 Size-dependent fracture of silicon nanoparticles during lithiation. ACS Nano6, 1522-1531.

(doi:10.1021/nn204476h)

22. Gu, M. et al. 2012 In situ TEM study of lithiation behavior of silicon nanoparticles attached to and embedded in a carbon matrix. ACS Nano6, 8439-8447.

(doi:10.1021/nn303312m)

23. Hertzberg, B., Alexeev, A. \& Yushin, G. 2010 Deformations in Si-Li anodes upon electrochemical alloying in nano-confined space. J. Am. Chem. Soc.132, 8548-8549. (doi:10.1021/ja1031997)

24. Bucci, G., Nadimpalli, S. P. V, Sethuraman, V. A., Bower, A. F. \& Guduru, P. R. 2014 Measurement and modeling of the mechanical and electrochemical response of amorphous Si thin film electrodes during cyclic lithiation. J. Mech. Phys. Solids62, 276-294.

(doi:10.1016/j.jmps.2013.10.005)

25. Bower, A. F., Guduru, P. R. \& Sethuraman, V. A. 2011 A finite strain model of stress, diffusion, plastic flow, and electrochemical reactions in a lithium-ion half-cell. J. Mech. Phys. Solids59, 804-828. (doi:10.1016/j.jmps.2011.01.003)

26. Zhao, K., Pharr, M., Cai, S., Vlassak, J. J. \& Suo, Z. 2011 Large plastic deformation in high-capacity lithium-ion batteries caused by charge and discharge. J. Am. Ceram. Soc.94, S226-S235. (doi:10.1111/j.1551-2916.2011.04432.x)

27. Purkayastha, R. T. \& McMeeking, R. M. 2012 An integrated 2-D model of a lithium ion battery: The effect of material parameters and morphology on storage particle stress. Comput. Mech.50, 209-227. (doi:10.1007/s00466-012-0724-8)

28. Di Leo, C. V., Rejovitzky, E. \& Anand, L. 2014 A Cahn-Hilliard-type phase-field theory for species diffusion coupled with large elastic deformations: Application to phaseseparating Li-ion electrode materials. J. Mech. Phys. Solids70, 1-29.

(doi:10.1016/j.jmps.2014.05.001) 
29. Chen, J., Liu, J., Qi, Y., Sun, T. \& Li, X. 2013 Unveiling the Roles of Binder in the Mechanical Integrity of Electrodes for Lithium-Ion Batteries. J. Electrochem. Soc.160, A1502-A1509. (doi:10.1149/2.088309jes)

30. Munao, D., Van Erven, J. W. M., Valvo, M., Garcia-Tamayo, E. \& Kelder, E. M. 2011 Role of the binder on the failure mechanism of Si nano-composite electrodes for Li-ion batteries. J. Power Sources196, 6695-6702. (doi:10.1016/j.jpowsour.2010.11.072)

31. Magasinski, A., Zdyrko, B., Kovalenko, I., Hertzberg, B., Burtovyy, R., Huebner, C. F., Fuller, T. F., Luzinov, I. \& Yushin, G. 2010 Toward efficient binders for Li-ion battery Si-based anodes: Polyacrylic acid. ACS Appl. Mater. Interfaces2, 3004-3010. (doi:10.1021/am100871y)

32. Sethuraman, V. A., Nguyen, A., Chon, M. J., Nadimpalli, S. P. V., Wang, H., Abraham, D. P., Bower, A. F., Shenoy, V. B. \& Guduru, P. R. 2013 Stress Evolution in Composite Silicon Electrodes during Lithiation/Delithiation. J. Electrochem. Soc.160, A739-A746. (doi:10.1149/2.021306jes)

33. 2013 ABAQUS Manual. In Dassault Systemes, Providence, RI.

34. Xu, R. \& Zhao, K. 2015 Mechanical interactions regulated kinetics and morphology of composite electrodes in Li-ion batteries. Extrem. Mech. Lett.

(doi:10.1016/j.eml.2015.10.004)

35. Di Leo, C. V., Rejovitzky, E. \& Anand, L. 2015 Diffusion-deformation theory for amorphous silicon anodes: The role of plastic deformation on electrochemical performance. Int. J. Solids Struct. (doi:10.1016/j.ijsolstr.2015.04.028)

36. Ding, N., Xu, J., Yao, Y. X., Wegner, G., Fang, X., Chen, C. H. \& Lieberwirth, I. 2009 Determination of the diffusion coefficient of lithium ions in nano-Si. Solid State Ionics. 180, 222-225. (doi:10.1016/j.ssi.2008.12.015)

37. Xie, J., Imanishi, N., Zhang, T., Hirano, A., Takeda, Y. \& Yamamoto, O. 2010 Li-ion diffusion in amorphous Si films prepared by RF magnetron sputtering: A comparison of using liquid and polymer electrolytes. Mater. Chem. Phys.120, 421-425. (doi:10.1016/j.matchemphys.2009.11.031)

38. Lee, S. W., McDowell, M. T., Choi, J. W. \& Cui, Y. 2011 Anomalous shape changes of silicon nanopillars by electrochemical lithiation. Nano Lett.11, 3034-3039. (doi:10.1021/nl201787r)

39. Liu, X. H. et al. 2011 Anisotropic swelling and fracture of silicon nanowires during lithiation. Nano Lett.11, 3312-3318. (doi:10.1021/nl201684d) 
40. Pharr, M., Suo, Z. \& Vlassak, J. J. 2014 Variation of stress with charging rate due to strain-rate sensitivity of silicon electrodes of Li-ion batteries. J. Power Sources270, 569575. (doi:10.1016/j.jpowsour.2014.07.153)

41. Nadimpalli, S. P. V., Tripuraneni, R. \& Sethuraman, V. a. 2015 Real-Time Stress Measurements in Germanium Thin Film Electrodes during Electrochemical Lithiation/Delithiation Cycling. J. Electrochem. Soc.162, A2840-A2846. (doi:10.1149/2.0941514jes)

42. Odgen, R. W. 1984 Non-Linear Elastic Deformations. New York: Dover.

43. Liu, Z. H., Maréchal, P. \& Jérôme, R. 1998 Blends of poly(vinylidene fluoride) with polyamide 6: interfacial adhesion, morphology and mechanical properties. Polymer (Guildf).39, 1779-1785. (doi:10.1016/S0032-3861(97)00222-X)

44. Fan, X.-D., Hsieh, Y.-L., Krochta, J. M. \& Kurth, M. J. 2001 Study on molecular interaction behavior, and thermal and mechanical properties of polyacrylic acid and lactose blends. J. Appl. Polym. Sci.82, 1921-1927. (doi:10.1002/app.2037)

45. Xu, Q. C., Ramachandran, A. R., Newnham, R. E. \& Tancrell, R. H. 1987 Proc. IEEE Ultrasonics Symposium. In Measurement of complex coefficients for thick PVDF polymer, pp. 663-666. Denver, Colorado.

46. Antonova, N. M. 2009 The mechanical properties of a composite coating with a polymer matrix based on sodium carboxymethylcellulose and aluminum powder. Russ. J. NonFerrous Met.50, 419-423. (doi:10.3103/S1067821209040221) 


\section{Figures}
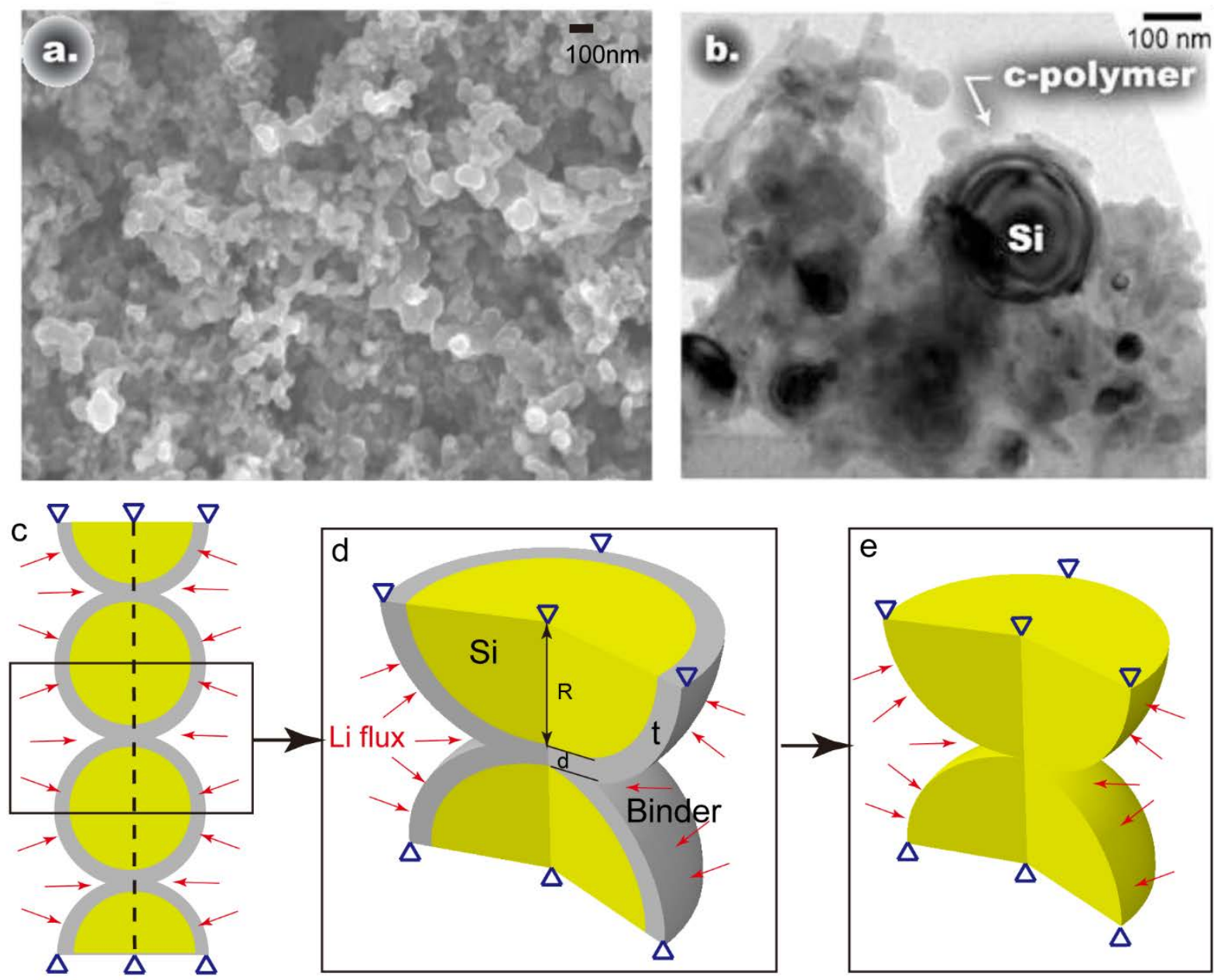

Figure 1. (a) SEM and (b) TEM images of Si-conductive polymer composite anode (Ref [22]). (c) Schematic drawing of aggregating Si-conductive polymer composite anode periodically aligning in one-dimension. Yellow represents Si particles, grey represents conductive polymer binder, red arrows indicate Li flux, and hollow triangles indicate vertical constraints. (d) Axisymmetric finite element model for Si-conductive polymer composite. $R, d$ and $t$ is the radius of Si particle, distance between neighboring particles and binder thickness, respectively. (e) Axisymmetric finite element model for contacting spherical Si particles without polymer binder $(t / R=0)$. 

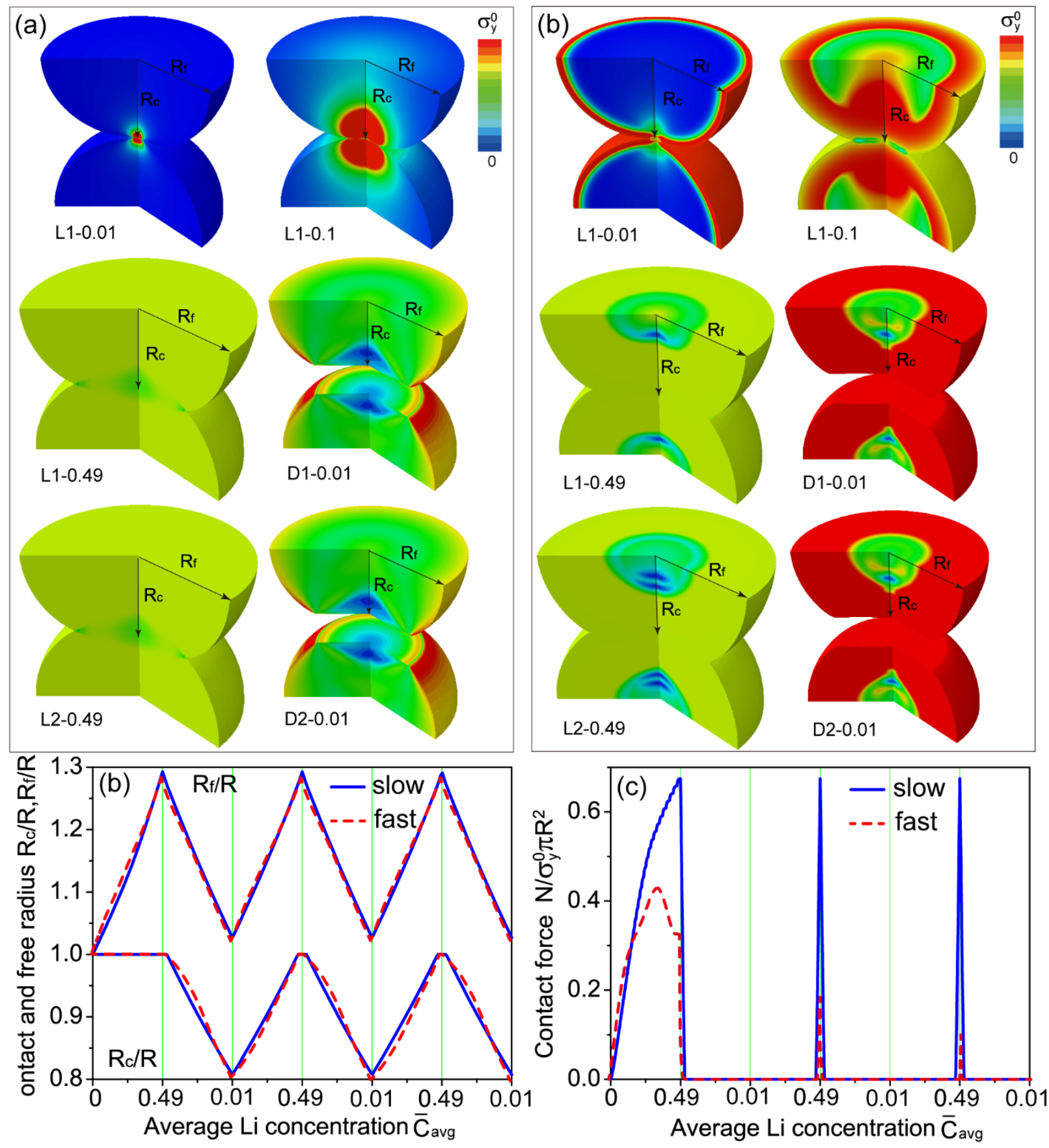

Figure 2. Shape change of contacting Si particles under galvanostatic lithiation (at very low rates) and with initial contact $(t / R=0)$ during the first three lithiation/delithiation cycles for varying lithiation/delithiation rate: (a) Shape sequences of contacting Si particles for slow lithiation/delithiation rate $I R / D C_{\max }=10^{-3}$; (b) Shape sequences of contacting Si particles for fast lithiation/delithiation rate $I R / D C_{\max }=10 ;\left(L_{1}\right.$ and $D_{1}$ represents the first lithiation and delithiation cycle, respectively. $L_{2}$ and $D_{2}$ represents the second lithiation/delithiation cycle, respectively. The contours show von Mises stress within the material, with the maximum level (red) corresponding to maximum yield stress $\sigma_{y}^{0}$ for crystal $\mathrm{Si}$, while the minimum level (blue) corresponds to a state of zero stress.) (c) Normalized radii 
free radius $\left(R_{f} / R\right)$ and contact radius $\left(R_{c} / R\right)$ as functions of average concentration. $R_{f}$ and $R_{c}$ represent free and contact radii; (d) Contact force between particles (normalized with maximum yield stress $\sigma_{y}^{0}=1.5 \mathrm{GPa}$ ) as function of average concentration. (Solid blue and dashed red curves correspond to the slow and fast lithiation/delithiation rates, respectively.)
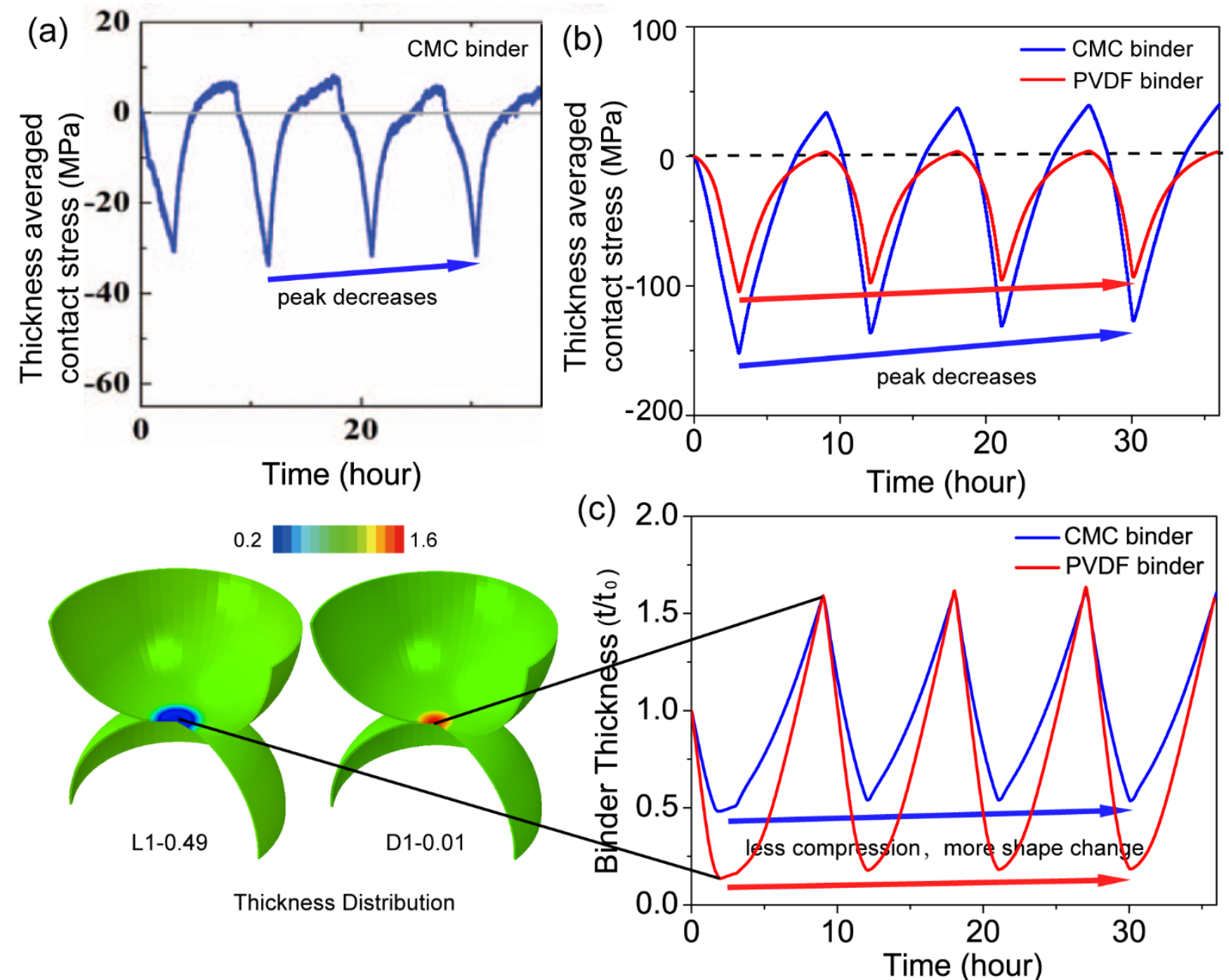

Figure 3. (a) Stress response of a Si/CMC composite electrode from an experiment in which the electrode was galvanostatically lithiated at $C / 50$ to a higher state-of charge during successive sets of cycles followed by galvanostatic delithiation. Delithiation was carried out at $\mathrm{C} / 100$ for each of the seven cycles until the potential reached $1.2 \mathrm{~V}$ vs. $\mathrm{Li} / \mathrm{Li}+$, followed by a potentiostatic hold at $1.2 \mathrm{~V}$ vs. $\mathrm{Li} / \mathrm{Li}+$ until the current decreased to less than $I=1 \mu \mathrm{A} / \mathrm{cm}^{2}$ [32]. (b) The evolution of thickness averaged contact stress $\mathrm{N} / 4 R^{2}$ between particles with CMC/PVDF binder under galvanostatic lithiation $\left(I R / D C_{\max }=0.116\right.$ for lithiation, $I R / D C_{\max }=0.058$ for delithiation, $t / R=$ 0.023) during the first four lithiation/delithiation cycles. (c) The evolution of binder thickness (normalized by the initial thickness) $t / t_{0}$ between Si particlesfor CMC/ PVDF binder. (Blue and red curves correspond to the CMC and PVDF binder, respectively.) The inserts are the thickness contoursof CMC binder forthe maximum compression and tension points in (c). Colors (blue to red) represent the normalized binder thicknesst $/ t_{0}(0.2-1.6)$. 

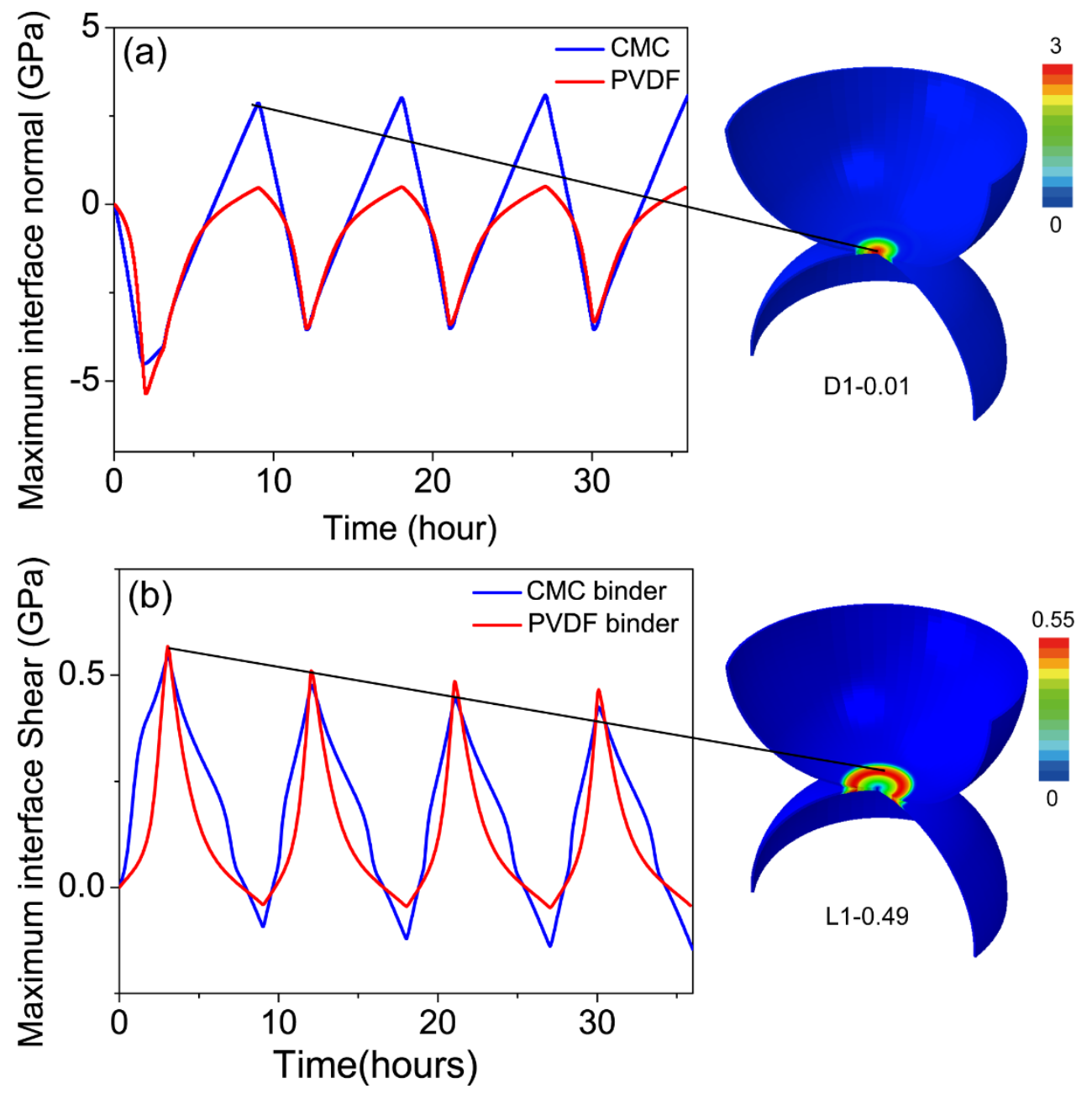

Figure 4: The evolution of maximum normal stress (a) and shear stress (b) on the interfaces of Si particles and CMC/PVDFbinder under galvanostatic lithiation $\left(I R / D C_{\max }=0.116\right.$ for lithiation, $I R / D C_{\max }=0.058$ for delithiation, $\mathrm{t} / \mathrm{R}=0.023$ ) during the first four lithiation/delithiation cycles. The inserts are the interface stress contours for fully charged and discharged states with maximum normal and shear interface stress for CMC binder. Colors (blue to red) represent normal stress (0-3GPa) or shear stress magnitude (0-0.55GPa). The interface debonding is predicted to occur first at these points with maximum normal and shear interface stress. 

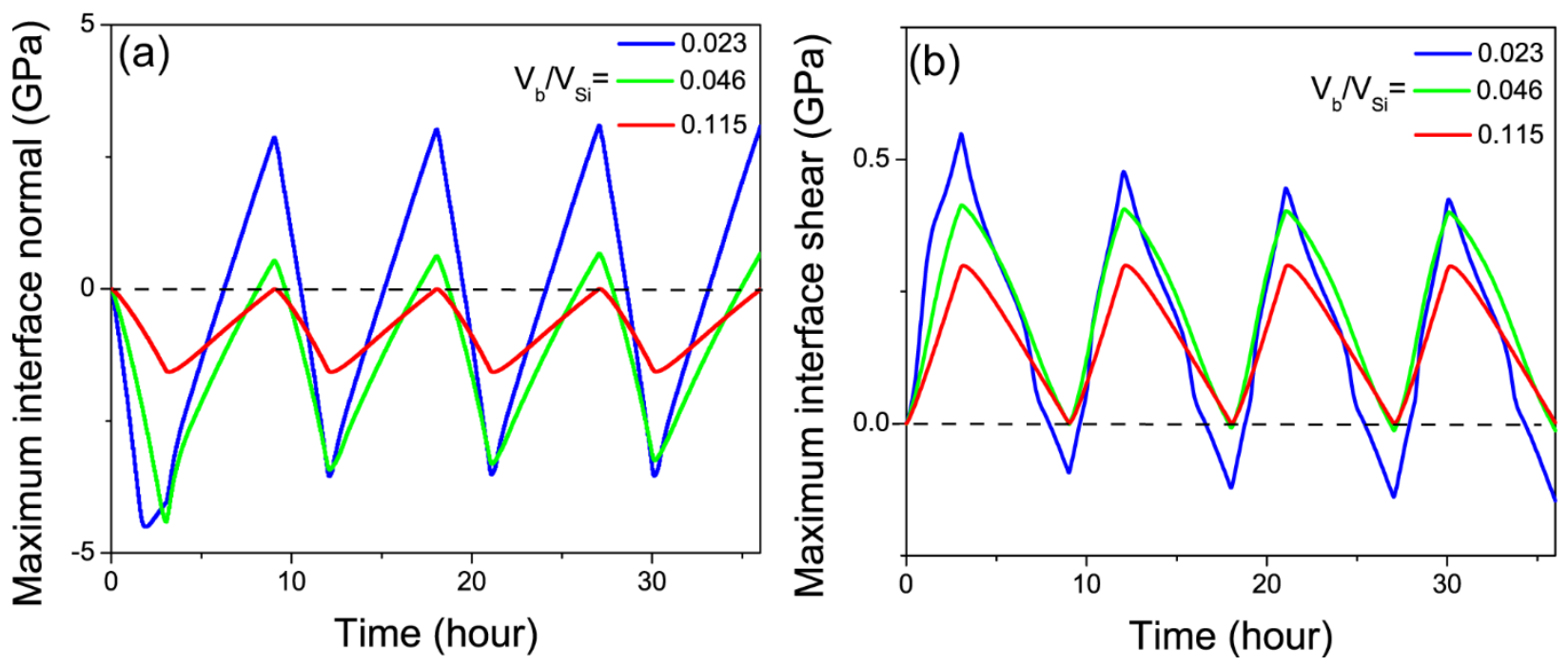

Figure 5: The evolution of maximum interface normal stress (a), and maximum interface shear stress (b) under galvanostatic lithiation $\left(I R / D C_{\max }=0.116\right.$ for lithiation, $I R / D C_{\max }=0.058$ for delithiation)on the interfaces of Si particles and CMC/PVDF binder under galvanostatic lithiation with various binder fraction $\left(\mathrm{V}_{b} / \mathrm{V}_{S i}=\right.$ $0.023,0.046,0.115)$ during the first four lithiation/delithiation cycles. 